\title{
ANSIEDAD ANTE EL APRENDIZAJE DEL INGLÉS COMO IDIOMA EXTRANJERO Y PERCEPCIÓN DE LAS ACTITUDES DEL MAESTRO DE INGLÉS EN ESTUDIANTES SALVADOREÑOS DE EDUCACIÓN SECUNDARIA
}

\section{ANXIETY ABOUT ENGLISH LEARNING AS A FOREIGN LANGUAGE AND THE PERCEPTION OF A TEACHER'S ATTITUDES BY SALVADORAN HIGH SCHOOL STUDENTS}

\author{
Delia Glomary España Reyes \\ Asociación Central Salvadoreña de la IASD, El Salvador \\ glomaryalf@hotmail.com \\ https://orcid.org/0000-0002-0233-2696
}

\begin{abstract}
RESUMEN
Se analizó la relación entre la ansiedad de estudiantes frente al aprendizaje del inglés y la percepción de la actitud del maestro, en un colegio privado de Quezaltepeque, El Salvador, en un estudio descriptivo, correlacional, no experimental y transversal. Participaron 86 alumnos de educación media y se utilizaron dos instrumentos, la Foreign Language Classroom Anxiety Scale (FLCAS) y una subescala de Percepción de Actitudes del Maestro de Inglés. Los resultados del estudio muestran un nivel medio de ansiedad. La percepción de la actitud del docente de inglés también obtuvo un nivel medio. Además, se encontró una correlación negativa significativa, pero débil entre ambos constructos; cuanto mejor es la percepción que tiene el estudiante de las actitudes del docente, se observa un menor puntaje de ansiedad. Se observó que las actitudes del docente percibidas por el estudiante están mayormente relacionadas con la ansiedad hacia los procesos y situaciones de aprendizaje. También se observó que la ansiedad hacia el aprendizaje del inglés tiende a disminuir con el avance del estudiante en el nivel de estudios.
\end{abstract}

Palabras clave: ansiedad, aprendizaje de lenguas extranjeras, actitudes docentes

\section{ABSTRACT}

The relationship between student anxiety about learning English and his/her perception of teacher's attitude was analyzed in a private school in Quezaltepeque, El Salvador, in a descriptive, correlational, non-experimental and cross-sectional study. Eighty-six high school students participated and two instruments were used, the Foreign Language Classroom Anxiety Scale (FLCAS) and a subscale of Perception of Attitudes of the English Teacher. The results of the study show a medium level of anxiety. The perception of the attitude of the English teacher also obtained an average level. 


\section{ESPAÑA REYES}

In addition, a significant but weak negative correlation was found between both constructs; the better the student's perception of the teacher's attitudes, the lower the anxiety score is observed. It was observed that the teacher's attitudes perceived by the student are mostly related to anxiety towards learning processes and situations. It was also observed that anxiety towards learning English tends to decrease as the student progresses in studies.

Keywords: English as a foreign language, anxiety about learning foreign languages, teaching attitudes

\section{Introducción}

En El Salvador, el estudio del idioma inglés como lengua extranjera se lleva a cabo por medio de una materia obligatoria que se imparte durante tres horas de clase a la semana, desde el séptimo hasta el undécimo grado en instituciones públicas. El plan de estudios del aprendizaje del inglés para la escuela secundaria se centra en el desarrollo del lenguaje con competencias integrales esenciales para comunicarse e interactuar correctamente. Su integración garantiza la adquisición de competencias esperadas, tales como la producción oral, la comprensión lectora, la expresión escrita y la comprensión auditiva del segundo idioma (Ministerio de Educación, 2008).

Sin embargo, el desarrollo de estas competencias se ve afectado por la ansiedad del estudiante ante el aprendizaje y su actitud hacia su maestro de inglés, pues se ha observado que los estudiantes con ansiedad tienen dificultades para hablar y comprender los sonidos y las estructuras del idioma (Yan y Horwitz, 2008).

Surgió un interés por esta temática entre investigadores y educadores, quienes pretenden explicar el efecto de la ansiedad en el nivel de aprendizaje de los estudiantes. Al respecto, Pichette (2009) señala que algunos estudiantes muestran menos confianza y están más propensos a la ansiedad que otros, ya que tienden a sentirse incómodos en la clase de inglés al participar en presencia de sus compañeros o cuando se enfrentan a realizar diferentes tareas académicas, puesto que se preocupan por cometer errores y sentirse avergonzados, por temor a ser criticados, a obtener una evaluación negativa o a exponerse a observaciones juiciosas, entre otras situaciones.

Entre los diversos factores afectivos que influyen en el aprendizaje de un idioma extranjero, la ansiedad aparece como un factor crucial que a menudo afecta la producción oral que se necesita para aprender, pues al no participar oralmente en los salones de clase, los estudiantes obtienen bajos logros y un bajo rendimiento (Melouah, 2013).

El aprendizaje del idioma inglés es fundamental en un mundo globalizado, de manera que dominarlo es muy importante hoy en día, pues los que tienen desarrolladas habilidades lingüísticas en este idioma tienen mejores oportunidades de trabajo y desarrollo.

\section{Ansiedad en el aprendizaje de una lengua extranjera}

Un factor que afecta a los estudiantes durante el proceso de aprendizaje es la ansiedad que se desarrolla en materias que les resultan difíciles, entre las cuales se encuentra el aprendizaje del 
idioma inglés como lengua extranjera. Seguramente, por este motivo existen diversas investigaciones que se centran en el estudio de la ansiedad hacia esta materia, que es denominada por la literatura como ansiedad ante el aprendizaje de lenguas extranjeras.

Respecto a lo anterior, Braun (2005) declaró que la psicología divide a la ansiedad en dos clases: (a) la ansiedad característica, la cual ya es parte de la personalidad de cada individuo y (b) el estado de ansiedad, que está relacionado con circunstancias específicas o situaciones determinadas, tales como miedo a hablar inglés frente a una clase, temor a no ser competente para interactuar con hablantes nativos y sensaciones de ansiedad ante la reprobación de un examen. Por esta razón, la ansiedad ha sido identificada como un factor importante que afecta el aprendizaje de idiomas extranjeros (Liu, 2012).

Horwitz et al. (1986) definieron la ansiedad como "el sentimiento subjetivo de tensión, aprehensión, nerviosidad y preocupación asociada con una excitación del sistema nervioso autónomo" (p. 125). Así como la ansiedad impide a algunas personas desarrollarse con éxito en ciencia o en matemáticas, también muchas personas encuentran el aprendizaje de lenguas extranjeras particularmente estresante, sobre todo en situaciones en el salón de clase.

De acuerdo con Ehrman (1996), la ansiedad puede interferir con la capacidad de usar habilidades específicas y necesarias para el aprendizaje de la lengua extranjera; asimismo, la ansiedad está relacionada con la interacción con otros, como lo es el caso de los alumnos que se sienten inhibidos acerca de su actuación, como, por ejemplo, al hablar en clase, porque se sienten juzgados por otros o inseguros de poder cumplir con las expectativas de su audiencia.

MacIntyre (1995) afirma que la ansiedad juega diferentes roles en el proceso de aprendizaje y puede facilitar o debilitar el rendimiento. Zheng (2008), por su parte, expresa que el aprendizaje de un idioma extranjero algunas veces puede ser una experiencia traumática para muchos alumnos, ya que son numerosos los estudiantes que manifiestan ansiedad. En consonancia con lo anterior, los resultados de Liu y Jackson (2008) mostraron que la mayor parte de los alumnos experimentan ansiedad ante el aprendizaje de una lengua extranjera.

Causas de ansiedad. Dentro de esta perspectiva, la ansiedad sobre el aprendizaje de lenguas extranjeras es atribuida a diferentes causas que son identificadas por Horwitz et al. (citado en Zheng, 2008), tales como "expresión oral, miedo a ser evaluado negativamente y ansiedad ante los exámenes" ( $p$. 127). Igualmente, Price (1991) concluyó que el nivel de dificultad de las clases de idioma extranjero, la percepción personal de la aptitud lingüística, ciertas variables de personalidad (como perfeccionismo y miedo de hablar en público) y experiencias estresantes en el salón de clases, son posibles causas de ansiedad.

Asimismo, Young (citado en Yan y Horwitz, 2008) identificó seis fuentes potenciales de ansiedad: (a) asuntos personales e interpersonales, (b) creencias del alumno acerca del aprendizaje del idioma, (c) creencias del profesor acerca de la enseñanza del lenguaje, (d) interacción entre maestro y alumno, (e) métodos en el salón de clase y (f) exámenes. Estas derivan de tres aspectos: el alumno, el maestro y la práctica instruccional.

Awan et al. (2010) indicaron también que una de las causas más grandes 


\section{ESPAÑA REYES}

de ansiedad es hablar frente a otros en un segundo idioma, seguida por las preocupaciones acerca de los errores gramaticales, pronunciación y el ser incapaz de hablar espontáneamente. De igual forma, sugieren que el ambiente del salón de clases debe ser animador y motivante y que los docentes necesitan tratar con cuidado las situaciones que provocan ansiedad.

\section{Actitud hacia el maestro}

Gkonou (2013) declara que el maestro constituye una parte integral del salón de clase; sin embargo, este, sin darse cuenta, despierta ansiedad en los estudiantes en una forma variada. Por ejemplo, un maestro con una actitud sentenciosa ya ha sido reportado como una de las causas más destacadas de ansiedad. Igualmente, las técnicas inapropiadas de corregir los errores y las críticas fuertes cuando se cometen equivocaciones en el transcurso de una lección probaron ser desalentadoras para los estudiantes y aumentaron la cantidad de miedo a evaluaciones negativas de parte del profesor.

Con respecto a la corrección de errores en el proceso de aprendizaje, Barrios Espinosa (1997) manifiesta el reconocimiento de que existe un cierto orden natural de adquisición que deberá llevar al docente a eludir una corrección excesiva de equivocaciones en el alumno, para evitar la ansiedad innecesaria que pueda producir.

Nugent (2009) sugiere que, al crear un sentido de bienestar en las relaciones maestro-alumno, los docentes pueden motivar a sus alumnos durante el proceso de aprendizaje. Por lo tanto, una relación positiva entre maestro y alumno es crucial dentro del salón de clase. Debido al impacto que esta relación podría tener, es necesario que los maestros entiendan que los estudiantes necesitan sentirse cómodos y en confianza con la persona con quien están aprendiendo. Esto significa que los maestros deben estar conscientes del impacto de la dimensión emocional en el salón de clase de inglés.

La investigación aquí reportada buscó explorar la relación entre la ansiedad que presenta el estudiante ante el aprendizaje del inglés y la actitud del maestro percibida por estudiantes del nivel medio de un colegio privado en Quezaltepeque, El Salvador, con el propósito de evaluar y realizar cambios, si fuera necesario, en el proceso de aprendizaje del inglés.

\section{Metodología \\ Tipo de investigación}

La presente investigación utilizó un abordaje cuantitativo, descriptivo, correlacional, no experimental y transversal.

\section{Participantes}

La población estuvo conformada por todos los alumnos del bachillerato de un colegio privado en Quezaltepeque, El Salvador. Estos tenían entre 16 y 18 años y estaban matriculados en el ciclo escolar 2018. Los estudiantes de esta población recibieron por tres horas semanales la materia de lenguaje extranjero (inglés) durante 40 semanas.

En el presente estudio no se realizó muestreo porque se estudiaron todos los elementos de la población, la cual se agrupó como se detalla en la Tabla 1.

\section{Tabla 1}

Distribución de la población de estudio por secciones

\begin{tabular}{lc}
\hline \multicolumn{1}{c}{ Nivel } & $n$ \\
\hline Primer año de bachillerato general & 45 \\
Segundo año de bachillerato general & 41 \\
Total & 86 \\
\hline
\end{tabular}




\section{Instrumentos}

Ansiedad en el estudiante de inglés. Para medir la ansiedad de los estudiantes frente al aprendizaje del inglés, se utilizó la versión en español de la escala Foreign Language Classroom Anxiety Scale (FLCAS). Esta se ha usado en un gran número de proyectos de investigación (Horwitz, 2001), pues es confiable y válida (Aida, 1994; Cheng et al., 1999). La FLCAS incluye 33 ítems de los cuales 20 se centran en las destrezas de comprensión y expresión oral. Los ítems restantes están relacionados con la ansiedad general ante el aprendizaje de la lengua.

Este instrumento, de acuerdo con Taboada et al. (citados en Hita Pedrosa y Fernández Martín, 2013), comprende las siguientes dimensiones: (a) aprensión comunicativa (13 ítems), (b) ansiedad ante los procesos y situaciones de aprendizaje de la LE (nueve ítems), (c) seguridad en el uso de la lengua extranjera dentro del aula (cuatro ítems), (d) seguridad en el uso de la lengua extranjera fuera del aula (tres ítems) y (e) actitudes negativas hacia el aprendizaje (cuatro ítems).

Cada ítem se valora conforme a una escala Likert con puntuaciones comprendidas entre 1 (totalmente en desacuerdo) y 5 (totalmente de acuerdo). En su versión española, la FLCAS obtuvo una consistencia interna de .89 y una correlación test-retest de .90 obtenida en un intervalo de ocho semanas (Pérez Paredes y Martínez Sánchez, 2001). Del mismo modo, la versión original obtuvo una consistencia interna de .93 y una correlación test-retest de .83 obtenida en un intervalo de ocho semanas (Horwitz, 1986; Horwitz et al., 1986).

Actitudes del maestro percibidas por el alumno. Para medir la percepción estudiantil de las actitudes del maestro de inglés, se utilizaron las 14 preguntas de la subescala Attitudes towards English Language Teacher incluidas en un instrumento más abarcante, de 67 ítems, en un estudio (Alrabai, 2014) de la percepción de 826 estudiantes conducido con el propósito de conocer el comportamiento de variables motivacionales del alumno, tales como intensidad motivacional, confianza en sí mismo, motivación intrínseca, actitudes hacia el profesor de inglés, ansiedad del lenguaje y otras. Cada ítem se valora de acuerdo con una escala Likert que va desde totalmente en desacuerdo (1) hasta totalmente en acuerdo (5).

El coeficiente de confiabilidad alfa de Cronbach fue de .88 en los ítems del 17 al 30 en la variable actitudes hacia el maestro de inglés. La versión en español aplicada en esta investigación fue validada con la asesoría de un experto en una prueba piloto con 21 estudiantes, teniendo como resultado un coeficiente de confiabilidad alfa de Cronbach de .817 .

\section{Análisis de datos}

El coeficiente de correlación $r$ de Pearson se utilizó para determinar la relación entre la ansiedad del estudiante frente al aprendizaje del inglés y la actitud del maestro percibida por el estudiante.

\section{Análisis descriptivo}

\section{Resultados}

La ansiedad en general mostró una media de $3.24(D E=0.704)$. Esto indica un nivel de ansiedad medio (56\%). Respecto a los factores, la aprensión comunicativa alcanzó la media más alta (ver Tabla 2). Las actitudes negativas hacia el aprendizaje resultaron con la media más baja. 


\section{ESPAÑA REYES}

Tabla 2

Descriptivos para los factores de la ansiedad $(\mathrm{N}=86)$

\begin{tabular}{llc}
\hline \multicolumn{1}{c}{ Factor } & $M$ & $D E$ \\
\hline Aprensión comunicativa & 3.4 & 0.890 \\
Ansiedad ante los procesos y situaciones de aprendizaje & 3.3 & 0.813 \\
Seguridad en el uso de la lengua extranjera dentro del aula & 3.3 & 0.909 \\
Seguridad en el uso de la lengua extranjera fuera del aula & 3.0 & 1.177 \\
Actitudes negativas hacia el aprendizaje & 2.6 & 0.953 \\
\hline
\end{tabular}

Respecto a la actitud del docente percibida por el alumno, se encontró una media de $3.3(D E=0.864)$. Esto indica una actitud media, donde se alcanza un promedio aritmético del $58 \%$.

\section{Análisis correlacional}

El coeficiente de correlación $r$ de Pearson mostró una correlación significativa, negativa y de baja importancia $(r=-.324, p=.002)$. Es decir, cuanto mayor puntaje en la actitud percibida del docente, se observa un menor puntaje de ansiedad. Los estudiantes que perciben una mejor actitud de sus docentes tienden a presentar menores niveles de ansiedad.

En la Tabla 3 se observan las correlaciones de los diferentes factores de ansiedad con la actitud y que la actitud negativa hacia el aprendizaje y la ansiedad ante los procesos y situaciones de aprendizaje son las que muestran mayor índice de correlación.

\section{Tabla 3}

Correlaciones entre los factores de ansiedad y la actitud

\begin{tabular}{lcc}
\hline & \multicolumn{2}{c}{ Actitud } \\
\cline { 2 - 3 } Factor de ansiedad & $r$ & $p$ \\
\hline Seguridad en el uso de la lengua extranjera dentro del aula & -.164 & .132 \\
Aprensión comunicativa & -.235 & .030 \\
Actitudes negativas hacia el aprendizaje & -.360 & .001 \\
Ansiedad ante los procesos y situaciones de aprendizaje & -.369 & .000 \\
Seguridad en el uso de la lengua extranjera fuera del aula & -.020 & .853 \\
\hline
\end{tabular}

\section{Otros hallazgos}

No se encontró diferencia significativa de medias de las variables y sus factores según el género. Por otro lado, se encontró diferencia significativa de ansiedad $\left(t_{(84)}=2.272, p=\right.$ .026) y aprensión comunicativa $\left(t_{(84)}\right.$ $=2.401, p=.019)$ entre las secciones. En ambos casos, los estudiantes de primer año muestran niveles más altos de ansiedad que los estudiantes de segundo año.

\section{Discusión}

Según los resultados de este estudio, la actitud del docente percibida por el alumno está significativamente correlacionada con la ansiedad hacia el aprendizaje del inglés. Es decir, los estudiantes que percibieron una mejor actitud 
de parte de sus docentes en la forma de enseñanza y evaluación, en aspectos que tienen que ver específicamente con la aprensión comunicativa y las situaciones de aprendizaje, presentaron menores niveles de ansiedad.

De acuerdo con Braun (2005), los cursos de lenguas extranjeras provocan reacciones de ansiedad específicas que pueden obstruir o impedir el proceso de adquisición de una lengua extranjera. Esto sugiere la necesidad de que los docentes de lenguas extranjeras sean conscientes de que el proceso de aprendizaje involucra no solo aspectos lingüísticos y cognitivos, sino también aspectos afectivos, ya que existen técnicas que pueden ser utilizadas para ayudar a reducir los niveles de ansiedad que los alumnos experimentan en el aula.

Al respecto, Thibaut et al. (2018) confirman que la conducta de una persona está fuertemente influenciada por sus actitudes, además de que la conducta del profesor juega un rol fundamental en sus prácticas en el salón de clases. Es por ello que las actitudes de los maestros están significativamente relacionadas con el aspecto afectivo de sus estudiantes durante el desarrollo de cada una de sus clases.

En el presente estudio, la ansiedad manifiesta un nivel medio ante los procesos y situaciones de aprendizaje, en coincidencia con Falagán Carbajo (2016), quien también identificó elementos de este factor como los que causan mayor porcentaje de ansiedad entre los estudiantes. Se trata de aspectos que se refieren a no entender lo que les dice el profesor y a cometer errores al expresarse en la lengua extranjera.

En consonancia con los resultados de esta investigación con respecto al nivel medio de ansiedad de los alumnos (56\%), los resultados de Pérez Fernández de las Heras (2015) indican que la mayor parte de los alumnos experimentan ansiedad ante el aprendizaje de una lengua extranjera, con una media del $52 \%$, muy parecida al resultado derivado de esta investigación.

En resumen, es primordial una relación positiva entre el docente y el alumno durante el proceso de aprendizaje del idioma inglés, debido a la impresión que esta relación puede causar. Por lo tanto, es necesario que el maestro comprenda la necesidad del estudiante de sentirse cómodo y con mayor confianza hacia la persona con la que está aprendiendo.

\section{Referencias}

Aida, Y. (1994). Examination of Horwitz, Horwitz, and Cope's construct of foreign language anxiety:

The case of students of Japanese. The Modern Language Journal, 78(2), 155-167. https://doi. org/10.2307/329005

Alrabai, F. (2014). Motivational practices in English as a foreign language classes in Saudi Arabia:

Teachers beliefs and learners perceptions. Arab World English Journal, 5(1), 224-246. https://www. awej.org/images/AllIssues/Volume5/Volume5Number1March2014/18.pdf

Awan, R. N., Azher, M., Anwar, M. N. y Naz, A. (2010). An investigation of foreign language classroom anxiety and its relationship with student's achievement. TLC: Journal of College Teaching and Learning, 7(11), 33-40. https://doi.org/10.19030/tlc.v7i11.249

Barrios Espinosa, M. E. (1997). Motivación en el aula de lengua extranjera. Encuentro: Revista de Investigación e Innovación en la Clase de Idiomas, 9, 17-30. http://www3.uah.es/encuentrojournal/ index.php/encuentro/issue/download/21/12 


\section{ESPAÑA REYES}

Braun, E. N. (2005). El rol de la ansiedad en el aprendizaje de lenguas extranjeras. Anuario de la Facultad de Ciencias Humanas, 7, 245-250. http://www.biblioteca.unlpam.edu.ar/pubpdf/anuario_ fch/n07a19braun.pdf

Cheng, Y., Horwitz, E. K. y Schallert, D. L. (1999). Language anxiety: Differentiating writing and speaking components. Language Learning, 49(3), 417-446. https://doi.org/10.1111/0023-8333.00095

Ehrman, M. E. (1996). Understanding second language learning difficulties. SAGE.

Falagán Carbajo, P. (2016). La ansiedad como factor decisivo en el aprendizaje de una segunda lengua [Tesis de maestría, Universidad de Oviedo]. Repositorio de la Universidad de Oviedo. http://digibuo .uniovi.es/dspace/handle/10651/38552

Gkonou, C. (2013). A diary study on the causes of English language classroom anxiety. International Journal of English Studies, 13(1), 51-68. https://doi.org/10.6018/ijes/2013/1/134681

Hita Pedrosa, M. L. y Fernández Martín, M. P. (2013). Ansiedad en L2 en un contexto de inmersión temprana. EduPsykhé: Revista de Psicología y Educación, 12(2), 151-165. https://journals.ucjc.edu/ EDU/article/view/3879/2825

Horwitz, E. K. (1986). Preliminary evidence for the reliability and validity of a Foreign Language Anxiety Scale. TESOL Quarterly: Teachers of English to Speakers of Other Languages, 20(3), 559564. https://doi.org/10.2307/3586302

Horwitz, E. K. (2001). Language anxiety and achievement. Annual Review of Applied Linguistic, 21, 112126. https://doi.org/10.1017/s0267190501000071

Horwitz, E. K., Horwitz, M. B. y Cope, J. (1986). Foreign language classroom anxiety. Modern Language Journal, 70(2), 125-132. https://doi.org/10.2307/327317

Liu, H. (2012). Understanding EFL undergraduate anxiety in relation to motivation, autonomy and language proficiency. e-FLT: Electronic Journal of Foreign Language Teaching, 9(1), 123-139. http:/e-flt.nus.edu.sg/v9n12012/liu.pdf

Liu, M. y Jackson, J. (2008). An exploration of Chinese EFL Learners' unwillingness to communicate and foreign language anxiety. The Modern Language Journal, 92(1), 71-86. https://doi.org/10.1111/ j.1540-4781.2008.00687.x

MacIntyre, P. D. (1995). How does anxiety affect second language learning? A reply to Sparks and Ganschow. The Modern Language Journal, 79(1), 90-99. https://doi.org/10.2307/329395

Melouah, A. (2013). Foreign language anxiety in EFL speaking classrooms: A case study of first-year LMD students of English at Saad Dahlab University of Blida, Algeria. Arab World English Journal, 4(1), 64-76. https://www.awej.org/index.php/volume-4-2013/32-awej-volume-4-number-1-2013/182-asma-melouah

Ministerio de Educación. (2008). English syllabus tenth and eleventh grades high school.

Nugent, T. T. (2009). The impact of teacher-student interaction on student motivation and achievement [Tesis doctoral, University of Central Florida]. STARS. https://stars.library.ucf.edu/etd/3860

Pérez Fernández de las Heras, A. I. (2015). La ansiedad en el aprendizaje de lenguas extranjeras en educación primaria: un estudio pseudo-longitudinal [Tesis de maestría, Universidad de Cantabria]. Repositorio Abierto de la Universidad de Cantabria. http://hdl.handle.net/10902/7841

Pérez Paredes, P. y Martínez Sánchez, F. (2001). A Spanish version of the Foreign Language Classroom Anxiety Scale: Revisiting Aida's factor analysis. RESLA: Revista Española de Lingüística Aplicada, 14, 337-352. https://dialnet.unirioja.es/servlet/ articulo?codigo $=871315$

Pichette, F. (2009). Second language anxiety and distance language learning. Foreign Language Annals, 42(1), 77-93. https://doi.org/10.1111/j.1944-9720.2009.01009.x

Price, M. L. (1991). The subjective experience of foreign language anxiety: Interviews with highly anxious students. En E. K. Horwitz y D. J. Young (Eds.), Language anxiety: From theory and research to classroom implications (pp. 101-108). Prentice Hall. 


\section{ANSIEDAD ANTE EL APRENDIZAJE DEL INGLÉS}

Thibaut, L., Knipprath, H., Dehaene, W. y Depaepe, F. (2018). The influence of teachers's attitudes and school context on instructional practices in integrated STEM education. Teaching and Teacher Education, 71, 190-205. https://doi.org/10.1016/j.tate.2017.12.014

Yan, J. X. y Horwitz, E. K. (2008). Learners' perceptions of how anxiety interacts with personal and instructional factors to influence their achievement in English: A qualitative analysis of EFL learners in China. Language Learning, 58(1), 151-183. https://doi.org/10.1111/j.1467-9922.2007.00437.x

Zheng, Y. (2008). Anxiety and second/foreign language learning revisited. Canadian Journal for New Scholars in Education, 1(1), 1-12. https://journalhosting.ucalgary.ca/index.php/cjnse/article/ view/30393

Recibido: 27 de abril de 2020

Revisado: 28 de junio de 2020

Aceptado: 6 de julio de 2020 